msh-mss Mathématiques et sciences humaines

200 | Hiver 2012

Hommage à Marc Barbut

\title{
Les recherches de Marc Barbut sur la mesure des inégalités
}

Marc Barbut's research on the measure of inequality

Jesús Basulto et José Maria Arribas

\section{OpenEdition}

1 Journals

Édition électronique

URL : http://journals.openedition.org/msh/12361

DOI : $10.4000 / \mathrm{msh} .12361$

ISSN : 1950-6821

Éditeur

Centre d'analyse et de mathématique sociales de l'EHESS

Édition imprimée

Date de publication : 13 décembre 2012

Pagination : p. 49-66

ISBN : 09876936

ISSN : 0987-6936

Référence électronique

Jesús Basulto et José Maria Arribas, "Les recherches de Marc Barbut sur la mesure des inégalités », Mathématiques et sciences humaines [En ligne], 200 | Hiver 2012, mis en ligne le 13 décembre 2012, consulté le 23 juillet 2020. URL : http://journals.openedition.org/msh/12361 ; DOI : https://doi.org/ $10.4000 / \mathrm{msh} .12361$ 


\title{
LES RECHERCHES DE MARC BARBUT SUR LA MESURE DES INÉGALITÉS
}

\author{
Jesús BASULTO ${ }^{1}$, José Maria ARRIBAS ${ }^{2}$
}

\begin{abstract}
RÉSUMÉ - La dernière publication de Marc Barbut en Espagne "The income distribution. A theory of Fréchet" (2010) nous a permis de revisiter quelques moments clé des recherches de Marc Barbut à propos de la modélisation et la mesure des inégalités de Pareto. Marc Barbut [2003(a)] discute de deux propriétés des lois stables établies par Lévy et liées au modèle de Pareto. Marc Barbut (2010) a généralisé le modèle de distribution du revenu proposé par Fréchet [1939], composé d'une fonction potentielle pour les revenus faibles et moyens, et du modèle de Pareto pour les revenus élevés. Marc Barbut [1998] a utilisé ces moyennes conditionnelles pour caractériser le modèle II de Pareto et Marc Barbut [2003(b)] propose une reformulation de la discussion entre Pareto et Sorel.
\end{abstract}

MOTS-CLÉS - Marc Barbut, Distribution des revenus, Mesure des inégalités

ABSTRACT - Marc Barbut's research on the measure of inequality

Marc Barbut's last publication in Spain: "The income distribution. A theory of Fréchet" (2010) let us review some of his key-research points related to the modeling and the measurement of Pareto inequalities. Marc Barbut [2003(a)] is interested in the extensions of Pareto's income distribution model I. From P. Lévy, Barbut notes two properties of the stable laws related to Pareto's model. Barbut (2010) generalized an income distribution model proposed by Fréchet [1939] which is formed by a potential function for low and medium incomes and by Pareto's model for the high incomes. Barbut [1998] used those conditional means to characterize Pareto's model II and Barbut [2003(b)] proposes a reformulation of the discussion between Pareto and Sorel.

KEYWORDS - Marc Barbut, Income distribution, Measure of inequality

Les travaux de Marc Barbut sur la mesure des inégalités et ses réflexions sur Pareto, Sorel, Levy et Fréchet ont servi d'inspiration à différentes recherches sur Pareto et sur l'indice de concentration de Gini à l'Université de Séville ${ }^{3}$, que nous prolongeons ci-dessous.

\footnotetext{
${ }^{1}$ Departamento de Economía aplicada, Universidad de Sevilla, basulto@us.es

2 Departamento de Sociología I, Universidad Nacional de Educación a Distancia, Madrid, jarribas@poli.uned.es

3 J. Basulto et J.J. Busto (2010), “Gini's Concentration Ratio (1908-1914)” et J. Basulto, J. J. Busto et R. Sánchez (2011), "El Concepto de Desigualdad en Vifredo Pareto (1848.1923)”.
} 


\section{SUR UNE INTERPRÉTATION DU PARAMÈTRE $\alpha$ DU MODÈLE I DE PARETO}

Marc Barbut passa beaucoup de son temps de recherche aux discussions sur l'interprétation du paramètre $\alpha$ du modèle I de Pareto ${ }^{4}$. L'article publié dans la revue Empiria "Ideología, matemáticas y ciencias sociales : V. Pareto, G. Sorel y la ambiguedad en la comparación de las desigualdades" est peut-être un des meilleurs supports pour résumer le débat.

G. Sorel, proposait dans les pages de son article «Le devenir social» [1897], l'interprétation suivante du paramètre $\alpha$ :

J'appelle nombre de personnes ayant un revenu donné le nombre des gens ayant un revenu compris dans un intervalle relativement constant autour $d u$ chiffre considéré : ainsi, je prendrai, par exemple, les nombres des revenus compris entre 4900 et 5100, francs - entre 9800 et 10200 - puis entre 14700 et 15300, etc..., chaque tranche a une épaisseur relative constante. D'après la loi de Bernoulli ces écarts se traduisent, à peu près, par des estimations égales. Je compare ensuite les nombres de revenus compris entre deux tranches successives : je suppose qu'il y ait, par exemple, 8000 personnes ayant des revenus compris entre 4900 et 5100 et 6000 ayant des revenus dans la tranche suivante : je dirai que sur 8000 candidats à l'ascension, 6000 seulement arrivent à atteindre l'échelon supérieur, que 2000 ont échoué et que par suite la difficulté de l'ascension est mesurée par le rapport de 2000 à 8000 .

Si on traite la question par le calcul infinitésimal, on trouve que la loi de M. Pareto revient à cette interprétation : "La difficulté pour l'ascension est constante et mesurée par la quantité $\alpha$ » [Sorel, 1897, p. 587].

Voyons une explication de texte :

Pour le modèle I de Pareto

$$
1-F_{X}(x)=\bar{F}_{X}(x)=\left(\frac{h}{x}\right)^{\alpha}, \text { pour } x \geq h<0 \text { et } \alpha>0,
$$

considérons la transformation $Y=\operatorname{Ln}(X)$ qui lie le revenu à son logarithme népérien. Sorel, d'après Daniel Bernoulli, interprète les valeurs de $Y$ comme utilités ${ }^{5}$ des revenus de $X$, la fonction de distribution de la nouvelle variable aléatoire $Y$ est :

$$
\bar{F}_{Y}(y)=\bar{F}_{X}\left(e^{y}\right)=\frac{h^{\alpha}}{e^{y \alpha}} .
$$

Avec $\bar{F}_{Y}\left(y_{h}\right)=\bar{F}_{X}(h)=1$, où $y_{h}=\operatorname{Ln}(h)$ est le minimum de la variable $Y$, il résulte que sa fonction de distribution est :

$$
\bar{F}_{Y}(y)=\bar{F}_{X}\left(e^{y}\right)=\frac{e^{y_{h} \alpha}}{e^{y \alpha}}=e^{-\left(y-y_{h}\right)^{\alpha}}
$$

\footnotetext{
${ }^{4}$ La loi de Pareto affirme que les revenus supérieurs à une valeur donnée $x$ se trouvent dans la proportion d'environ $\left(\frac{h}{x}\right)^{\alpha}$ - Marc Barbut utilise la notation $\left(\frac{x_{0}}{x}\right)^{\alpha}$ - et l'exposant $\alpha$, qui était compris entre 1 et 2 dans tous les cas étudiés empiriquement par Pareto, est un paramètre essentiel dans la discussion de M. Barbut.

5 «L'utilité est une mesure du bien être ou de la satisfaction obtenue par la consommation, ou du moins l'obtention d'un bien ou d'un service », (Daniel Bernoulli, La théorie sur la mesure du risque, 1738).
} 
Nous voyons donc que $Y$ suit un modèle exponentiel avec des paramètres $\left(y_{h}, \alpha\right)$.

$\mathrm{Si}$, dans le parcours de la variable aléatoire $Y$, nous sélectionnons trois valeurs successives $y_{i}<y_{i+1}<y_{i+2}$, telles que $y_{i+1}-y_{i}=y_{i+2}-y_{i+1}=\lambda$, c'est-à-dire telles que la différence entre ces valeurs est constante et égale à $\lambda>0$, les revenus qui correspondent aux utilités $y_{i}<y_{i+1}<y_{i+2}$, sont, respectivement, $e^{y_{i}}<e^{y_{i+1}}<e^{y_{i+2}}$, où $\frac{e^{y_{i+1}}}{e^{y_{i}}}=\frac{e^{y_{i+2}}}{e^{y_{i+1}}}=e^{\lambda}$. Nous voyons que des paires de valeurs de $Y$ avec des différences égales correspondent à des paires de valeurs de $X$ avec des quotients égaux.

Nous allons maintenant calculer ce que Sorel appelle «la difficulté de l'ascension », c'est-à-dire l'expression suivante :

$$
q_{i+1 \mid i}=\left[1-\frac{\bar{F}_{Y}\left(y_{i+1}\right)-\bar{F}_{Y}\left(y_{i+2}\right)}{\bar{F}_{Y}\left(y_{1}\right)-\bar{F}_{y}\left(y_{i+1}\right)}\right]
$$

Dans l'expression (1), la différence $\bar{F}_{Y}\left(y_{i+k}\right)-\bar{F}_{y}\left(y_{i+1+k}\right)$ est la proportion d'individus avec des utilités de revenus comprises dans l'intervalle $\left[y_{i+k}, y_{i+1+k}\right)$, pour $k=0,1$.

L'expression (1) est la probabilité conditionnelle qu'un individu qui a son utilité de revenu compris dans l'intervalle $\left[y_{i}, y_{i+1}\right)$ n'atteigne pas l'intervalle $\left[y_{i+1}, y_{i+2}\right)$. La formule (1) exprime la diminution des individus passant de l'intervalle $\left[y_{i}, y_{i+1}\right)$ à l'intervalle $\left[y_{i+1}, y_{i+2}\right)$, par rapport aux individus de l'intervalle $\left[y_{i}, y_{i+1}\right)$. Si, par exemple, le premier intervalle contient 8000 individus et le second 6000 individus, l'expression (1) vaut $\frac{8000-6000}{8000}=0,25$, c'est-à-dire que le nombre d'individus du second intervalle a diminué de $25 \%$ par rapport aux individus du premier intervalle.

Sorel interprète cette formule (1) en disant que :

Il faut bien observer que la notion d'ascension n'est pas donnée pour la statistique; elle est ajoutée pour l'interprétation; on assimile les revenus à des corps mobiles ayant un mouvement déterminé et éprouvant plus ou moins de difficultés à se mouvoir : comme on n'a pas de moyens pour calculer les forces, on ne sait rien de la direction et de la nature de ce mouvement. Dans nos sociétés, il existe certainement une classe qui prospère et absorbe la plus grande partie des revenus ; c'est toujours d'elle qu'on parle quand on raisonne sur la richesse nationale: dans son sein il y a une ascension et celle-ci peut se concilier avec la déchéance du plus grand nombre de manière à produire une apparente ascension nationale. C'est de ce mouvement fictif d'enrichissement que traitent les économistes dans leurs apologies de la société capitaliste [Sorel, 1897, p. 588].

Un modèle dynamique de changement social qui conduit à la loi de Pareto a été proposé par Champernowne [1953].

Si nous divisons la formule (1) par l'amplitude commune aux intervalles d'utilités considérées soit $\lambda$, nous avons l'expression :

$$
\frac{q_{i+1 \mid i}}{\lambda}=\left[1-\frac{\bar{F}_{Y}\left(y_{i+1}\right)-\bar{F}_{Y}\left(y_{i+2}\right)}{\bar{F}_{Y}\left(y_{i}\right)-\bar{F}_{Y}\left(y_{i+1}\right)}\right]\left(\frac{1}{\lambda}\right),
$$

qui est la densité de probabilité conditionnelle. 
En développant l'expression (2), nous avons :

$$
\frac{q_{i+1 \mid i}}{\lambda}=\frac{\frac{\bar{F}_{Y}\left(y_{i}\right)-2 \bar{F}_{Y}\left(y_{i+1}\right)+\bar{F}_{Y}\left(y_{i+2}\right)}{\lambda^{2}}}{\frac{\bar{F}_{Y}\left(y_{i}\right)-\bar{F}_{y}\left(y_{i+1}\right)}{\lambda}} .
$$

$\mathrm{Si}$, dans l'expression (3), nous substituons les utilités $y_{i}<y_{i+1}<y_{i+2}$ par $y<y+\lambda<y+2 \lambda$ et que, de plus, nous prenons leur limite quand $\lambda \rightarrow 0$, nous obtenons, en utilisant les définitions de la première et seconde dérivée d'une fonction, le résultat suivant :

$$
-\frac{\bar{F}_{Y}^{\prime \prime}(y)}{\bar{F}_{Y}^{\prime}(y)}
$$

Si nous posons que (4) est une constante égale au paramètre $\alpha$, il en résulte une équation différentielle qui a comme solution générale le modèle exponentiel $\bar{F}_{Y}(y)=e^{-\left(u-u_{h}\right)^{\alpha}} \quad$ [J.S. Chipman, 1976]. Nous voyons ainsi que la condition $-\frac{\bar{F}_{Y}^{\prime \prime}(y)}{\bar{F}_{Y}^{\prime}(y)}=\alpha$ caractérise le modèle I de Pareto.

Si, dans (3) nous substituons $F_{Y}(y)=e^{-\left(y-y_{h}\right)^{\alpha}}$, c'est-à-dire que nous supposons que le revenu se distribue selon le premier modèle de Pareto, il en résulte l'expression suivante :

$$
\frac{1-2 e^{-\alpha \lambda}+e^{-2 \alpha \lambda}}{\left[1-e^{-\alpha \lambda}\right] \lambda}
$$

Et, en prenant la limite de (5), quand $\lambda \rightarrow 0 \mathrm{~s}$, on obtient :

$$
\lim _{\lambda \rightarrow 0} \frac{1-2 e^{-\alpha \lambda}+e^{-2 \alpha \lambda}}{\left[1-e^{-\alpha \lambda}\right] \lambda}=\alpha
$$

Le résultat (6) permet d'interpréter le paramètre $\alpha$. En effet, pour $\lambda$ suffisamment petit nous avons l'approximation $\frac{q_{i+1 \mid i}}{\lambda} \cong \alpha$ : quand la probabilité conditionnelle $q_{i+1 \mid i}$ augmente (diminue) alors le paramètre $\alpha$ augmente (diminue). Si nous utilisons la probabilité conditionnelle complémentaire, c'est-à-dire, $p_{i+1 \mid i}=1-q_{i+1 \mid i}$, le résultat de Sorel apporte une interprétation du paramètre $\alpha$, montrant que quand ce paramètre diminue (augmente) alors le nombre d'individus d'un intervalle d'utilité de revenu augmente (diminue) et passent à l'intervalle suivant, de plus grande de utilité.

\section{LES LOIS STABLES DE PAUL LÉVY ET LE MODÈLE DE PARETO}

Dans son article «Homme moyen ou homme extrême ? De Vilfredo Pareto (1896) à Paul Lévy (1936) en passant par Maurice Fréchet et quelques autres (2003)», Marc Barbut étudie les connexions entre les résultats obtenus par ces auteurs. 
Rappelons, d'après W. Feller [1966, chap.6, v.II], la définition de la distribution stable. Une distribution $\mathbf{R}$ est stable si les variables $X, X_{1}, X_{2}, \ldots, X_{n}$, indépendantes les unes des autres et ayant la même distribution $\mathbf{R}$, vérifient, pour tout $n$, que la somme $S_{n}=X_{1}+X_{2}+\ldots+X_{n}$ a bien la même distribution que la variable $c_{n} X+\gamma_{n}$, où $c_{n}>0$. Le modèle normal est un exemple de distribution stable.

Un premier résultat est la relation entre la première loi de Pareto et les théorèmes limites proposés par Paul Lévy pour définir les lois stables avec les paramètres $0<\alpha<2$ et $\beta=1$. $^{6}$

Reprenons ensuite la fonction de distribution d'un modèle stable avec $\alpha=0,7$ et $\beta=1$, avec un paramètre ayant une échelle de 3,646 et une origine nulle. Le calcul a été réalisé par simulation pour un échantillon de 500 observations de ce modèle stable, en utilisant le procédé de Chamber, Mallows et Stuck [1976]. Nous avons également calculé une autre approximation à ce modèle stable à partir du théorème limite suivant, proposé par P. Lévy.

Nous avons sélectionné 500 échantillons indépendants du modèle de Pareto, avec $\alpha=0,7$ et un revenu minimum $h=1$, de taille $n=20$. Pour chaque échantillon, nous avons calculé l'expression $\left(\sum_{k=1}^{n} x_{k}\right) n^{-\frac{1}{\alpha}}$, qui sont les valeurs qui s'approchent de la fonction de distribution stable.

Paul Lévy a démontré que l'expression $\left(\sum_{k=1}^{n} x_{k}\right) n^{-\frac{1}{\alpha}}$ converge vers une loi stable avec les paramètres $\alpha=0,7$ et $\beta=1$, quand $n$ tend vers l'infini [P. Lévy, 1937, p. 203].

Dans le graphique suivant, sont présentées les deux fonctions de distribution calculées :

Graphique 1

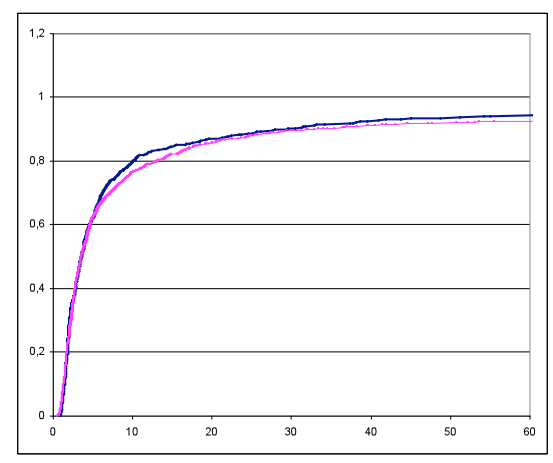

Le Graphique 1 montre que l'approximation proposée par Paul Lévy (la courbe située ci-dessous) s'approche bien de la fonction de distribution stable.

Un autre résultat lié à Pareto est que les lois stables sont asymptotiquement parétiennes, c'est-à-dire que, quand la valeur de la variable tend vers l'infini, la fonction

\footnotetext{
${ }^{6}$ Nous avons pris $\beta=1$ et $\alpha$ entre $0<\alpha<1$ parce que nous voulons travailler avec une variable stable positive, ce qui nous permet de le rattacher aux distributions de revenus proposées par Pareto. Le paramètre $\beta$ varie entre -1 et 1 . Une variable stable est symétrique quand $\beta=0$.
} 
de distribution stable s'approche de la fonction du modèle I de Pareto. Le graphique suivant compare les fonctions de distribution stable $\alpha=0,7, \beta=1$, ayant une échelle de 3,646 et une origine nulle, à la fonction de distribution de Pareto avec $\alpha=0,7$ et revenu minimum $h=1$. Nous montrons les probabilités cumulées plus élevées que 0,49 :

Graphique 2

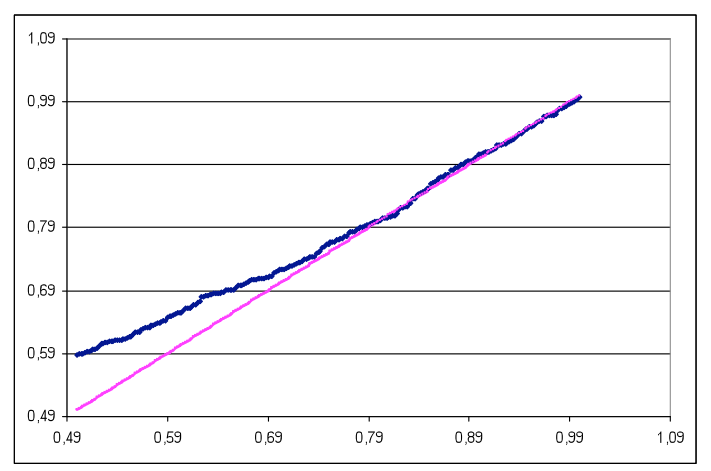

Dans le Graphique 2, nous observons le rapprochement des deux courbes à partir de la probabilité cumulée de 0,79 , c'est-à-dire pour l'extrémité à droite avec une probabilité de 0,21 .

Pour l'exemple traité, si la distribution $G$ est stable avec les paramètres $0<\alpha<1$ et $\beta=1$, Feller démontre que $x^{\alpha}[1-G(x)] \rightarrow \frac{1}{\Gamma(1-\alpha)}$, quand $x \rightarrow \infty$ (Chapitre 13). Ce résultat montre que, dans l'extrémité droite de la distribution stable, l'expression $[1-G(x)] \rightarrow 1-F(x)$, où $1-F(x) \infty \frac{1}{x^{\alpha}}$ quand $x \rightarrow \infty$, s'approche de la distribution de Pareto avec $0<\alpha<1$.

\section{3. "THE INCOME DISTRIBUTION: A THEORY OF FRECHET"7}

Dans la Section 1 de cet article, Marc Barbut rend compte de l'existence d'un dossier à l'Académie des sciences de Paris (boîte F-10) avec des travaux et manuscrits de Maurice Fréchet réalisés entre 1930 et 1960. À l'intérieur de ce dossier apparaissent les recherches que Fréchet réalisa sur la distribution des revenus et quelques essais sur l'amélioration de la Loi de Pareto. Des trois travaux cités dans la bibliographie sur Fréchet, nous allons nous centrer sur le travail intitulé «Sur les formules de répartition des revenus », publié en 1939 par la Revue de l'Institut international de statistique 3, (p. 321-338).

Dans ce travail, Fréchet propose un modèle pour la distribution du revenu qui peut être appliqué à tous les niveaux de revenus. Il complète de cette manière le modèle de Pareto qui, comme nous le savons, concerne seulement les revenus à partir d'une valeur élevée. Nous verrons que Marc Barbut propose un modèle pour la distribution du revenu qui couvre tous les niveaux de revenus et qui généralise le modèle de Fréchet.

\footnotetext{
${ }^{7}$ Barbut M. [2010], “The income distribution. A theory of Frechet”, Boletin de estadística e Investigación Operativa, Vol. 26(3), p. 218-237.
} 
Avant de présenter le résultat principal de Fréchet, nous allons lier le modèle exponentiel $\bar{F}_{Y}(y)=e^{-y}$, pour $y \geq 0$, aux modèles de distribution du revenu de Pareto.

Considérons la transformation $y=\alpha \operatorname{Ln}(x+\alpha)+\beta x \operatorname{Ln}(10)+b$, où $\alpha>0, \beta \geq 0$, $-\infty<\alpha<+\infty$, et la variable aléatoire $X$ qui a comme fonction de distribution $1-F_{X}(x)=\bar{F}_{X}(x)$, telle que $\bar{F}_{Y}(y)=\bar{F}_{X}(x)$. De plus, il existe un revenu minimum $h>0$, tel que $\alpha \operatorname{Ln}(h+\alpha)+\beta x \operatorname{Ln}(10)+b=0$, avec $b=-\alpha \operatorname{Ln}(h+\alpha)-\beta h \operatorname{Ln}(10)$, où $h>-a$ quand $\alpha<0$. De plus, $\bar{F}_{Y}(0)=\bar{F}_{X}(h)=1$.

La fonction de distribution de $X$ est alors :

$$
1-F_{X}(x)=\bar{F}_{X}(x)=\bar{F}_{Y}(y)=e^{-y}=e^{-L n(x-h)^{\alpha}-\beta x L n(10)-b}
$$

c'est-à-dire :

$$
\bar{F}_{X}(x)=\frac{e^{-b} 10^{-\beta x}}{(x+\alpha)^{\alpha}}=\frac{10^{h \beta}(h+\alpha)^{\alpha}}{(x+\alpha)^{\alpha}} 10^{-\beta x}
$$

Si nous opérons avec le paramètre $\beta^{*}=\frac{\beta}{\log (e)}$ à la place de $\beta$, alors le modèle (7) s'écrit :

$$
\bar{F}_{X}(x)=\frac{e^{h \beta^{*}}(h+\alpha)^{\alpha}}{(x+\alpha)^{\alpha}} e^{-\beta^{*} x}
$$

Nous voyons que (7) est le troisième modèle de la distribution du revenu de Pareto. En prenant $\beta=0$ on obtient le second modèle de Pareto et si, de plus, $\alpha=0$ dans (7), il en résulte le premier modèle de la distribution du revenu de Pareto. Pour $\beta=0$, le modèle (7) a déjà été utilisé par D'Addario [1949] dans son travail "Ricerche sulla curva dei redditi", publié dans le Giornale degli Economista e Annali di Economia, 8, (1-2), 91-144. Ce fut F. Y. Edgeworth [1898] qui proposa la "method of translation" («méthode de translation »), qui cherche une fonction de distribution à partir d'une Loi des erreurs, la première ou bien la seconde Loi des erreurs de Laplace, et un changement de variable.

Voyons maintenant le travail de Fréchet. Fréchet va partir de la première loi des erreurs de Laplace, c'est-à-dire :

$$
\bar{F}_{Y}(y)=\frac{e^{-|y|}}{2}, \text { où }-\infty<y<+\infty
$$

et considère la transformation $y=\alpha \operatorname{Ln}(x-h)+b$, avec la variable aléatoire $X>h>0$.

En rappelant que, d'une part, quand $y>0$, alors $\int_{0}^{y} e^{-|t|}=\int_{0}^{y} e^{-t} d t=1-e^{-y}$ et, d'autre part, quand $y<0$, alors $\int_{0}^{y} e^{-|r|}=\int_{0}^{y} e^{\prime} d t=e^{y}-1$.

Voyons la fonction de distribution $\bar{F}_{X}(x)$, de la variable aléatoire $X$.

La fonction $y=\alpha \operatorname{Ln}(x-h)+b$ coupe l'axe des abscisses au point $x_{M}$ tel que $\alpha \operatorname{Ln}\left(x_{M}-h\right)+b=0$, avec $e^{-b}=\left(x_{M}-h\right)^{\alpha}$. 
Pour $x \geq x_{M}, \quad \bar{F}_{X}(x)=\bar{F}_{Y}(y)=\frac{1}{2}-\frac{1}{2}\left(1-e^{-y}\right)=\frac{1}{2} e^{-y}=\frac{e^{-b}}{2(x-h)^{\alpha}}=\frac{A}{(x-h)^{a}}$, où $A=\frac{e^{-b}}{2}=\frac{\left(x_{M}-h\right)^{\alpha}}{2}$. De plus, comme $\bar{F}_{X}\left(x_{M}\right)=\bar{F}_{Y}(0)=\frac{1}{2}$, alors $x_{M}$ est la médiane de la fonction de distribution $\bar{F}_{X}(x)$.

Pour $h<x \leq x_{M}, \quad F_{X}(x)=\frac{1}{2} \int_{-\infty}^{\mathrm{y}} e^{t} d t=\frac{e^{y}}{2}=\frac{e^{\operatorname{Ln}(x-h)^{\alpha}+b}}{2}=\frac{e^{b}}{2}(x-h)^{\alpha}=B(x-h)^{\alpha}$, où $B=\frac{e^{b}}{2}=\frac{1}{2\left(x_{M}-h\right)^{\alpha}}$.

La fonction de distribution de la variable aléatoire $X$ est

$$
F_{X}(x)=\left\{\begin{array}{l}
B(x-h)^{\alpha}, h \leq x \leq x_{M} \\
1-\frac{A}{(x-h)^{\alpha}}, x \geq x_{M}
\end{array}\right.
$$

Nous voyons que quand $x \geq x_{M}$, on obtient le second modèle de la distribution du revenu de Pareto. Si, de plus, nous supposons que $h=0$, alors nous trouvons le premier modèle de distribution du revenu de Pareto.

Voyons maintenant la fonction de densité de la fonction de distribution: $1-F_{X}(x)=\bar{F}_{X}(x)$.

Quand $x \geq x_{M}$, la fonction de densité de $X$ est $f_{X}(x)=\frac{A \alpha}{(x-h)^{\alpha+1}}$. Quand $h<x \leq x_{M}$, alors $f_{X}(x)=B \alpha(x-h)^{\alpha-1}$. Pour $\quad x=x_{M}$, il résulte que $\frac{A \alpha}{\left(x_{M}-h\right)^{\alpha+1}}=B \alpha\left(x_{M}-h\right)^{\alpha-1}$.

Cette fonction de densité a un mode à la valeur $x_{M}$, étant donné que ses dérivées sont :

- Pour $x \geq x_{M}, \frac{d f_{X}(x)}{d x}=-A \alpha \frac{(\alpha+1)(x-h)^{\alpha}}{(x-h)^{2(\alpha+1)}}$, qui est négative et, ainsi, décroissante.

La dérivée à droite de la médiane est égale à $-\frac{\alpha(\alpha+1)}{2\left(x_{M}-h\right)^{\alpha}}$.

- Pour $h<x>x_{M}, \frac{d f_{X}(x)}{d x}=B \alpha(\alpha-1)(x-h)^{\alpha-2}$ qui est positive chaque fois que $\alpha>1$, et ainsi, est une fonction croissante. La dérivée à gauche de la médiane est égale à $\frac{\alpha(\alpha+1)}{2\left(x_{M}-h\right)^{\alpha}}$. Nous voyons donc que la médiane $x_{M}$ est aussi le mode. Nous voyons que Fréchet propose un modèle de distribution du revenu qui a un mode coïncidant avec la médiane qui couvre tous les niveaux de revenus et dont l'extrémité droite est un modèle de Pareto. 
Voyons maintenant la Section 2 de l'article de Marc Barbut dans laquelle il construit une distribution du revenu pour tous ces niveaux à la manière de Fréchet.

La fonction de distribution que propose M. Barbut est :

$$
F_{X}(x)=\left\{\begin{array}{l}
B(x-h)^{\beta}, h \leq x \leq x_{M} \\
1-\frac{A}{(x+a)^{a}}, x \geq x_{M}
\end{array}\right.
$$

où $B=\frac{1}{2\left(x_{M}-h\right)^{\beta}}$ et $A=\frac{\left(x_{M}+\alpha\right)^{\alpha}}{2}$ sont des quantités positives. Le revenu minimum est $h>0$.

Comme $F_{X}\left(x_{M}\right)=\frac{1}{2}, x_{M}$ est la médiane. Si $\alpha>0$ alors nous verrons que $\beta>1$. De plus, si $-\infty<\alpha<+\infty$ et que $\alpha<0$, alors $x \geq x_{M}>-\alpha$.

La fonction de densité de ce modèle est :

$$
f_{X}(x)=\left\{\begin{array}{l}
B \beta(x-h)^{\beta-1}, h \leq x \leq x_{M} \\
\frac{A \alpha}{(x+\alpha)^{\alpha+1}}, x \geq x_{M}
\end{array}\right.
$$

Maintenant, nous devons exiger que $B \beta\left(x_{M}-h\right)^{\beta-1}=\frac{A \alpha}{\left(x_{M}+\alpha\right)^{\alpha+1}}$, qui équivaut à la condition $\frac{\alpha}{\beta}=\frac{x_{M}+\alpha}{x_{M}-h}$ (formule 2.11 de M. Barbut).

La première dérivée de la fonction de densité est :

$$
\frac{d f_{X}(x)}{d x}=\left\{\begin{array}{l}
B \beta(\beta-1)(x-h)^{\beta-2}, h \leq x \leq x_{M} \\
-\frac{A \alpha(\alpha+1)}{(x+\alpha)^{\alpha+2}}, x \geq x_{M}
\end{array}\right.
$$

Nous voyons que si nous voulons que la fonction de densité soit croissante quand $h \leq x \leq x_{M}$, il faut que $\beta>1$. L'autre branche de la fonction de densité, quand $x \geq x_{M}$ est décroissante, ces comportements conduisent à ce que la médiane $x_{M}$ soit, de plus, le mode de la fonction de densité de ce modèle proposé par Marc Barbut.

Le modèle (10) proposé par Marc Barbut peut être généré, comme nous l'avons vu avec Fréchet, à partir du modèle exponentiel (8) et d'une transformation de la forme suivante :

$$
y=\left\{\begin{array}{l}
\beta \operatorname{Ln}(x-h)+b_{2}, h \leq x \leq x_{M} \\
\alpha \operatorname{Ln}(x+\alpha)+b_{1}, x \geq x_{M}
\end{array}\right.
$$

où $x_{M}$ vérifie les conditions : $\beta \operatorname{Ln}\left(x_{M}-h\right)+b_{2}=0$ et $\alpha \operatorname{Ln}\left(x_{M}+\alpha\right)+b_{1}=0$ avec $A=\frac{e^{-b_{1}}}{2}$ et $B=\frac{e^{b_{2}}}{2}$. ̊̀ partir de $\frac{b_{1}}{b_{2}}=\frac{\alpha}{\beta} \frac{\operatorname{Ln}\left(x_{M}+\alpha\right)}{\operatorname{Ln}\left(x_{M}-h\right)}$ et de la formule $2.11 \mathrm{de}$ 
M. Barbut, il en résulte l'expression $\frac{b_{1}}{b_{2}}=\frac{\operatorname{Ln}\left(x_{M}+\alpha\right)^{\left(x_{M}+\alpha\right)}}{\operatorname{Ln}\left(x_{M}-h\right)^{\left(x_{M}-h\right)}}$ qui n'inclut pas les paramètres $\alpha$ et $\beta$.

Si Fréchet utilise une transformation pour toute l'échelle des revenus, Marc Barbut, lui, utilise des transformations différentes pour chacune des parties qui divisent cette échelle.

Un exemple de (11), avec $\alpha=3,42, \beta=3, \alpha=3$ et $h=10$ est :

Graphique 3

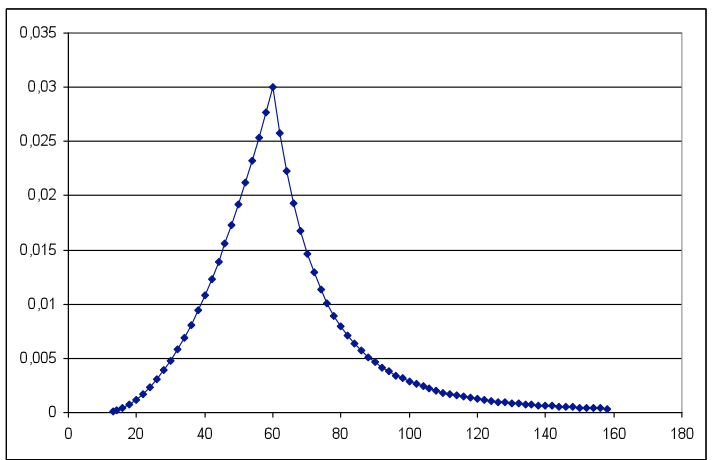

où la médiane, $x_{M}=60$, est aussi le mode. Nous comparons cette courbe de densité avec la courbe que Pareto recueille dans le graphique 51 de son Cours d'économie tome II, p. 315, où l'auteur théorise la forme que devrait avoir le segment ascendant de la courbe des revenus, affirmant que ce segment « serait probablement très écrasant » (dans notre cas, Pareto compare ce segment de la courbe avec le segment perpendiculaire à l'axe des abscisses qui coupe cette courbe à la médiane) ce que montre le Graphique 3.

\section{MOYENNES CONDITIONNELLES}

Dans un article de 1914, Bowley utilise la formule $M(x)=\frac{x \alpha}{\alpha-1}$, où $M(x)$ est le revenu moyen des revenus plus élevés que $x, x \geq h>0$, du modèle I de Pareto. Nous devons supposer que $\alpha>1$.

Bresciani-Turroni utilise dans un article de 1910 la formule suivante :

$$
R(x)-R(y)=\delta[x N(x)-y N(y)]
$$

où $R(x)$ et $N(x)$ sont le total, respectivement, des revenus et des individus, avec des revenus au-dessus de $x$, avec $\delta=\frac{\alpha}{\alpha-1}$.

Une expression équivalente à cette formule est :

$$
R(x)-\delta x N(x)=R(y)-\delta y N(y) .
$$


Quand $x=h$ avec $\mu=\frac{R(h)}{N(h)}=h \delta$, il en résulte que $R(y)-\delta y N(y)=0$. Par conséquent $M(x)=\frac{x \alpha}{\alpha-1}$. Nous voyons que Bresciani-Turroni aurait pu arriver à cette dernière formule.

Un calcul direct pour arriver à la formule $M(x)=\frac{x \alpha}{\alpha-1}$ est de partir de l'expression $R(x)=\frac{\alpha N(h) h^{\alpha}}{(\alpha-1) x^{a-1}}$ qui, en la divisant par $x N(x)=\frac{x N(h) h^{\alpha}}{x^{\alpha}}$ donne le résultat $\frac{M(x)}{x}=\delta$.

La condition pour laquelle $\frac{M(x)}{x}=\delta$, pour tout $x \geq h>0$ avec $\delta>1$, caractérise le premier modèle de Pareto avec $\alpha>1$. Voyons ce résultat :

D'abord, si nous supposons que la relation $\frac{M(x)}{x}=\delta$ équivaut à $\frac{R(x)}{x N(x)}=\delta$ et que, pour tout $x \geq h>0$, on a $\int_{x}^{\infty} t f(x) d x=\delta x \int_{x}^{\infty} f(x) d x$, en dérivant les deux limites de ces intégrales, il en résulte que $-x F^{\prime}(x)=\delta N(x)-\delta x F^{\prime}(x)$, où $F(x)$ est la fonction de distribution de $f(x)$ et $N(x)=1-F(x)$. Comme $\frac{d F(x)}{d x}=-\frac{d}{d x}(1-F(x))=-\frac{d N(x)}{d x}$, on obtient l'équation différentielle suivante $x N^{\prime}(x)=\delta N(x)+\delta x N^{\prime}(x)$, c'est-à-dire $\frac{N^{\prime}(x)}{N(x)}=-\frac{\delta}{x(\delta-1)}$. Les solutions de cette équation différentielle sont $\operatorname{Ln}(N(x))=-\frac{\delta}{\delta-1} \operatorname{Ln}(x)+\operatorname{Ln}(A)$. Ensuite $N(x)=\frac{A}{x^{\frac{\delta}{\delta-1}}}$, et en ajoutant la condition $N(h)=\frac{A}{h^{\frac{\delta}{\delta-1}}}$, il en résulte que $A=N(h) h^{\frac{\delta}{\delta-1}}$. Nous voyons que l'on obtient le modèle I de Pareto avec le paramètre $\alpha=\frac{\delta}{\delta-1}>1$.

Dans le travail «Une famille de distributions : des parétiennes aux contreparétiennes. Application à l'étude de la concentration urbaine et son évolution », Marc Barbut part de l'hypothèse : $M(x)=\beta x+h$, avec les conditions nécessaires $\beta \geq 0$ et $h \geq 0$, et obtient :

- $\operatorname{pour} \beta>1$ le modèle II de Pareto,

- pour $0 \leq \beta \leq 1$ les distributions qu'il appelle « contre-paretiennes », et

- pour $\beta=1$ le modèle exponentiel avec paramètres de localisation et d'échelle. 
Un critère fonctionnel d'inégalité est $M(x(p))=\frac{\int_{x(p)}^{\infty} t f(t) d t}{\int_{x(p)}^{\infty} f(t) d t}$, où $x(p)$ est le quantile pour $0<p<1$, un revenu en dessous de la proportion $p$ d'individus; et $M(x(p))$ est le revenu moyen pour les revenus au-dessus de $x(p)$. Dans le modèle I de Pareto avec $\alpha>1$, nous avons vu que $M(x(p))=x(p) \frac{\alpha}{\alpha-1}$, que nous pouvons écrire $M(x(p))=\frac{x(p)}{h} \frac{h \alpha}{\alpha-1}=\frac{x(p)}{h} \mu$, où $\mu=\frac{h \alpha}{\alpha-1}$ et $\frac{x(p)}{h}=(1-p)^{-\frac{1}{\alpha}}$.

Voyons comment se comporte ce critère fonctionnel quand nous comparons deux modèles distincts du modèle I de Pareto avec $\alpha>1$.

CAS I : $\alpha_{2}<\alpha_{1}, h_{2}>h_{1}$, alors $M_{2}\left(x_{2}(p)\right)>M_{1}\left(x_{1}(p)\right)$ pour tout $0<p<1$.

DÉMONSTRATION : $\alpha_{2}<\alpha_{1}, h_{2}>h_{1}$ équivaut à $\frac{\alpha_{2}}{\alpha_{2}-1}>\frac{\alpha_{1}}{\alpha_{1}-1} \quad$ et, aussi à $(1-p)^{-\frac{1}{\alpha_{2}}}>(1-p)^{-\frac{1}{\alpha_{1}}}$ et de l'inégalité $h_{2}>h_{1}$, il résulte que $\mu_{2}>\mu_{1}$ et, également $\frac{x_{2}(p)}{h_{2}}>\frac{x_{1}(p)}{h_{1}}$, par conséquent $M_{2}\left(x_{2}(p)\right)>M_{1}\left(x_{1}(p)\right), 0<p<1$. Si $\alpha_{2}=\alpha_{1}$ et $h_{2}>h_{1}$ ou si $\alpha_{2}<\alpha_{1}$ et $h_{2}=h_{1}$, on obtient aussi le même résultat. Exemple : $\alpha_{1}=1,8$, $h_{1}=24: \alpha_{2}=1,6, h_{2}=34$.

CAS II : $\alpha_{2}>\alpha_{1}$ et $\mu_{2}=\mu_{1}$, alors $h_{2}>h_{1}$ et $M_{2}\left(x_{2}(p)\right)<M_{1}\left(x_{1}(p)\right)$ pour tout $0<p<1$.

DÉMONSTRATION : Comme $h=\mu \frac{\alpha-1}{\alpha}$, de $\alpha_{2}>\alpha_{1}$, il résulte que $\frac{\alpha_{2}-1}{\alpha_{2}}>\frac{\alpha_{1}-1}{\alpha_{1}}$, puis $h_{2}>h_{1}$. De $\alpha_{2}>\alpha_{1}$, il résulte aussi que $\frac{x_{2}(p)}{h_{2}}<\frac{x_{1}(p)}{h_{1}}$, puis $M_{2}\left(x_{2}(p)\right)<M_{1}\left(x_{1}(p)\right)$ pour tout $0<p<1$. Exemple : $\alpha_{1}=\frac{4}{3}, h_{1}=20 ; \alpha_{2}=2, h_{2}=40$.

CAS III : $\alpha_{2}>\alpha_{1}$ et $\mu_{2}>\mu_{1}$, alors $h_{2}>h_{1}$ et les fonctions $M_{2}\left(x_{2}(p)\right)$ et $M_{1}\left(x_{1}(p)\right)$ se coupent en un point.

DÉMONSTRATION : $\alpha_{2}>\alpha_{1}$ équivaut à $\frac{\alpha_{2}-1}{\alpha_{2}}>\frac{\alpha_{1}-1}{\alpha_{1}}$ et, également, à $(1-p)^{-\frac{1}{\alpha_{2}}}<(1-p)^{-\frac{1}{\alpha_{1}}}$, et comme $\mu_{2}>\mu_{1}$, il résulte que $h_{2}>h_{1}$. De même, $\alpha_{2}>\alpha_{1}$ équivaut à $\frac{x_{2}(p)}{h_{2}}<\frac{x_{1}(p)}{h_{1}}$, comme $M(x(p))=\frac{x(p)}{h} \mu$, où $\mu_{2}>\mu_{1}$ et $\frac{x_{2}(p)}{h_{2}}<\frac{x_{1}(p)}{h_{1}}$, alors $M_{2}\left(x_{2}(p)\right)$ et $M_{1}\left(x_{1}(p)\right)$ se coupent en un point, étant donné que 
$M(x(p))=x(p) \frac{\alpha}{\alpha-1}$ est linéaire en $x(p)$, pour $0<p<1$. Exemple : $\alpha_{1}=1,3, h_{1}=20$; $\alpha_{2}=1,5, h_{2}=39$. Quand $p<0,947$, alors $M_{2}\left(x_{2}(p)\right)>M_{1}\left(x_{1}(p)\right)$, et quand $p \geq 0,947$, alors $M_{2}\left(x_{2}(p)\right)<M_{1}\left(x_{1}(p)\right)$.

CAS IV : $\alpha_{2}>\alpha_{1}$ et $\mu_{2}<\mu_{1}$, alors $M_{2}\left(x_{2}(p)\right)<M_{1}\left(x_{1}(p)\right)$ pour tout $0<p<1$.

DÉMONSTRATION : $\alpha_{2}>\alpha_{1}$ implique que $(1-p)^{-\frac{1}{\alpha_{2}}}<(1-p)^{-\frac{1}{\alpha_{1}}}$, et comme $M(x(p))=\frac{x(p)}{h} \mu$, où $\frac{x(p)}{h}=(1-p)^{-\frac{1}{\alpha}}$, il résulte que $M_{2}\left(x_{2}(p)\right)<M_{1}\left(x_{1}(p)\right)$ pour tout $0<p<1$. Exemple : $\alpha_{1}=1,3, h_{1}=20 ; \alpha_{2}=1,5, h_{2}=24$, où $h_{2}>h_{1}$; en changeant $h_{2}=19$, nous avons le cas $h_{2}<h_{1}$; enfin nous pouvons changer $h_{2}=20$, avec $h_{2}<h_{1}$.

Une application utilisée par G. Sorel est la relation entre le quantile $x(p)=h(1-p)^{-\frac{1}{\alpha}}$, quand $p=0,95$, c'est-à-dire $5 \%$ des individus avec des revenus audessus du quantile $x(0,95)$, et du revenu moyen de tous les revenus $x \geq h>0$. Cette relation est de la forme $\frac{x(0,95)}{\mu}=\frac{h(1-0,95)^{-\frac{1}{\alpha}}}{h \frac{\alpha}{\alpha-1}}=\frac{0,05^{-\frac{1}{\alpha}}}{\frac{\alpha}{\alpha-1}}$, soit une fonction qui dépend seulement du paramètre $\alpha>1$. Le tableau de Sorel [1897, p. 605] est le suivant :

Tableau [Sorel, 1897, p. 605]

\begin{tabular}{|c|c|c|c|c|c|c|}
\hline$\alpha$ & 2 & 1,70 & 1,50 & 1,40 & 1,30 & 1,20 \\
\hline$\frac{x(0,95)}{\mu}$ & 2,236 & 2,398 & 2,456 & 2,427 & 2,311 & 2,023 \\
\hline
\end{tabular}

Dans le tableau, on observe que la relation augmente d'abord jusqu'à un maximum pour ensuite diminuer au fur et à mesure qu'augmentent les valeurs de $\alpha$, avec $\alpha>1$. Marc Barbut [2007, chap. 10] calcule que le maximum de cette relation est 1,5010 .

Dans ce tableau, nous voyons que pour $5 \%$ des plus riches, le revenu minimum par rapport au revenu moyen de tous les revenus $x \geq h>0$, augmente jusqu'à 2,456, pour diminuer ensuite. Marc Barbut affirme qu' «Il n'en reste pas moins que dès que $\alpha>1,5010$, seuil de richesse et revenu moyen tendent à se rapprocher ; en ce sens, l'inégalité diminue quand $\alpha$ augmente ».

On démontre que $\frac{x(0,95)}{\mu}=\frac{M(x(0,95))}{M(\mu)}$, c'est-à-dire que la relation entre le revenu minimum des $5 \%$ les plus riches et le revenu moyen de toute la population est égal à la relation entre le revenu moyen des $5 \%$ les plus riches et le revenu moyen des revenus au-dessus du revenu moyen de la population. 
Si nous prenons le revenu moyen au-dessus du revenu moyen de la population, par rapport au revenu minimum $h$, c'est-à-dire $\frac{M(\mu)}{h}=\left[\frac{\alpha}{\alpha-1}\right]^{2}$, et que nous faisons la même chose avec le revenu moyen au-dessus du quantile $x(0,95)$, c'est-à-dire $\frac{M(x(0,95))}{h}=\frac{\alpha}{\alpha-1}(0,05)^{-\frac{1}{\alpha}}$, il en résulte le graphique suivant :

\section{Graphique 4}

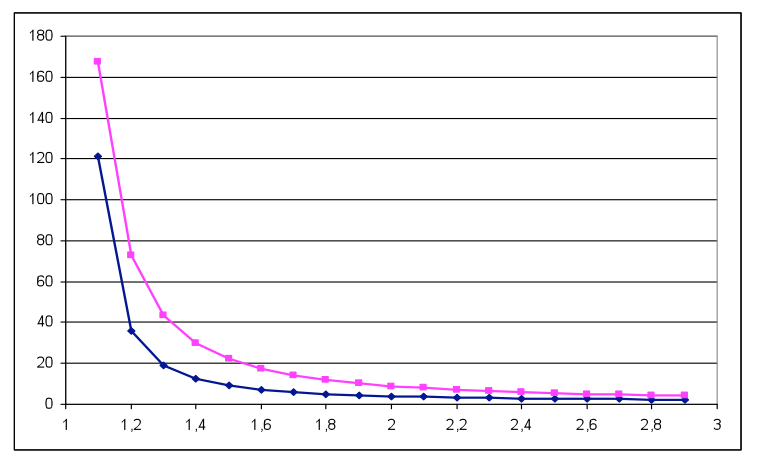

où la courbe supérieure est le revenu moyen, relatif au revenu minimum $h$, des revenus au-dessus du quantile $x(0,95)$; alors que la courbe inférieure est le revenu moyen, relatif au revenu minimum $h$, pour les revenus au-dessus de la moyenne de la population.

Nous avons vu que le quotient entre ces différents revenus est maximum quand $\alpha=1,5010$. La formule qui calcule ce maximum est : $\alpha_{\max }=\frac{\operatorname{Ln}(1-p)}{1-\operatorname{Ln}(1-p)}$, pour le quantile $x(p)$. Le quotient $\frac{x(0,95)}{\mu}=\frac{M(x(0,95))}{M(\mu)}$ vaut l'unité pour une valeur $\tilde{\alpha}$ telle que $\left(\frac{\tilde{\alpha}-1}{\tilde{\alpha}}\right)^{\tilde{\alpha}}=1-p$, pour $\tilde{\alpha}<\alpha_{\max }$, et pour $1-p=0,05$ on obtient la valeur approximative $\tilde{\alpha}=1,06361$. Nous voyons donc que quand $\alpha<\tilde{\alpha}$ alors $M(\mu)>M(x(0,95))$, c'est-à-dire la moyenne au-dessus de $\mu$ est plus élevée que la moyenne au-dessus du quantile $x(0,95)$, pour $\alpha=\tilde{\alpha}$ elles coïncident, et pour $\alpha>\tilde{\alpha}$ il résulte que $M(\mu)<M(x(0,95))$. Quand $\alpha \rightarrow \infty$, alors le quotient $\frac{M(x(0,95))}{M(\mu)} \rightarrow 1$. La courbe du quotient $\frac{M(x(0,95))}{M(\mu)}$ pour $1-p=0,05$ est : 
Graphique 5

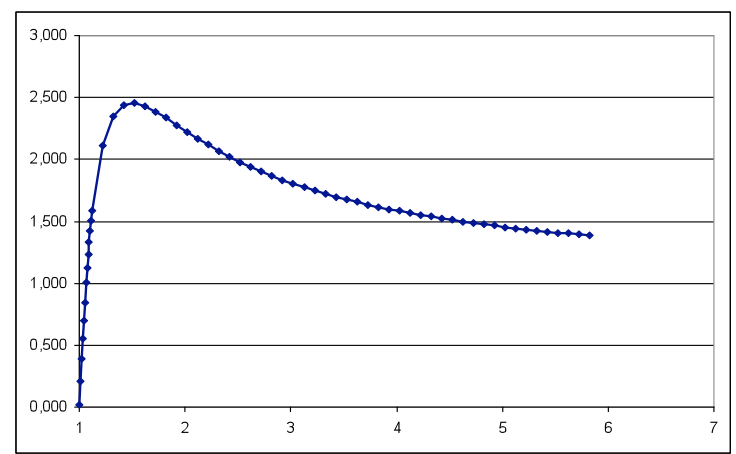

Ce graphique montre que la valeur maximum est obtenue pour $\alpha=1,5010$ et, pour $\alpha \rightarrow 1$, le quotient $\frac{M(x(0,95))}{M(\mu)}$ tend vers zéro et, finalement, quand $\alpha \rightarrow \infty$, alors $\frac{M(x(0,95))}{M(\mu)}$ tend vers 1 .

Maintenant nous pouvons donner une interprétation du Tableau de Sorel [1897, p. 605]. Si nous observons ce tableau depuis $\alpha>1$, nous voyons que le quotient $\frac{x(0,95)}{\mu}$ augmente jusqu'à une valeur maximum pour $\alpha=1,5010$, dans le tableau $\alpha=1,5$, pour décroître ensuite. Le tableau ne fait pas état que :

- pour $\alpha<\tilde{\alpha}$, avec $\alpha>1$, le quotient $\frac{x(0,95)}{\mu}$ est inférieur à l'unité, c'est-à-dire que la moyenne de la population dépasse la valeur minimum des $5 \%$ les plus riches ;

- pour $\alpha=\tilde{\alpha}$ la moyenne de la population et le revenu minimum des $5 \%$ les plus riches coïncident ;

- pour $\alpha>\tilde{\alpha}$, le revenu minimum des $5 \%$ les plus riches dépasse le revenu moyen de la population, sa différence diminuant au fur et à mesure que $\alpha>1$ augmente.

Nous voyons alors que pour des valeurs de $\alpha$ autour de $\tilde{\alpha}=1,06361$ ou bien très élevées le revenu minimum des $5 \%$ les plus riches est proche du revenu moyen de la population.

Voyons quelques graphiques :

EXEMPLE I : Quand $\alpha=\tilde{\alpha}, h=100$, il résulte que $\mu=x(0,95)=1671,91053$ et, également, $M(\mu)=M(p(0,95))=27952,84817$, avec $\frac{x(0,95)}{\mu}=1$. Nous obtenons la fonction de distribution, $1-F(x)$ du Modèle I de Pareto. 


\section{Graphique 6}

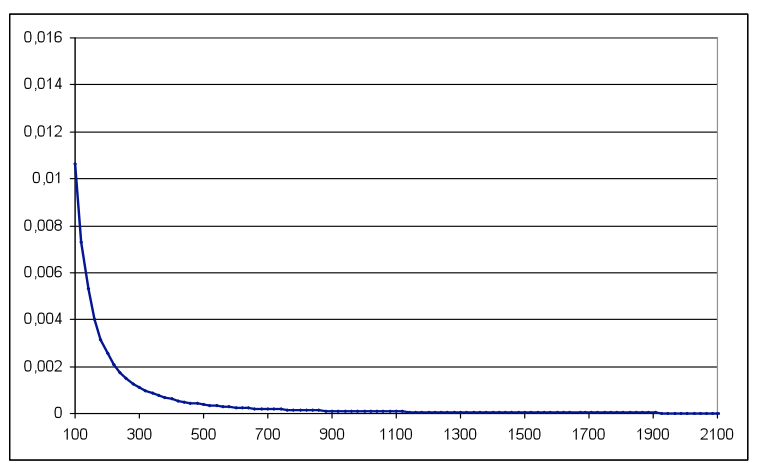

Le Graphique 6 montre les revenus situés dans l'intervalle $[100,2100]$ où le pourcentage des individus avec des revenus au-dessus de 2100 est 3,923\%.

EXEMPLE II : Pour une valeur de $\alpha=1,05, h=100$, on obtient : $\mu=2100$, $x(0,95)=1734,10818, \quad M(\mu)=44100 \quad$ et $\quad M(x(0,95))=36416,27174, \quad$ avec $\frac{x(0,95)}{\mu}=0,8257$. Nous obtenons la fonction de distribution, $1-F(x)$ du Modèle I de Pareto.

Graphique 7

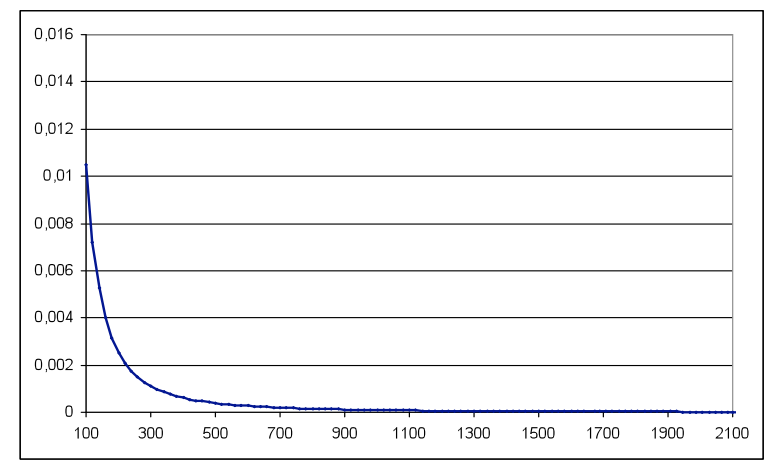

Le Graphique 7 montre les revenus situés dans l'intervalle [100, 2100], où le pourcentage d'individus avec des revenus au-dessus de 2100 est de 4,089 \%. En comparant cet exemple II à l'exemple I, nous voyons que, quand $\alpha$ diminue et $\mu$ augmente, alors $M(x(0,95))$ augmente, résultat recueilli dans le Cas IV des comparaisons de deux modèles distincts qui suivent le modèle I de Pareto.

EXEMPLE III : Enfin, pour une valeur de $\alpha=1,5, h=100$, on obtient : $\mu=300$, $x(0,95)=736,8063, \quad M(\mu)=900 \quad$ et $\quad M(x(0,95))=2210,418899, \quad$ avec $\frac{x(0,95)}{\mu}=2,4560$. Nous obtenons la fonction de distribution, $1-F(x)$ du Modèle I de Pareto. 
Le Graphique 8 montre les revenus situés dans l'intervalle [100, 2100] où le pourcentage d'individus avec des revenus au-dessus de 2100 est 1,039\%. En comparant cet exemple III avec l'exemple I, nous voyons que quand $\alpha$ augmente et $\mu$ diminue, alors $M(x(0,95))$ diminue, un résultat obtenu dans le Cas IV des comparaisons de deux modèles distincts qui suivent le modèle I de Pareto.

\section{Graphique 8}

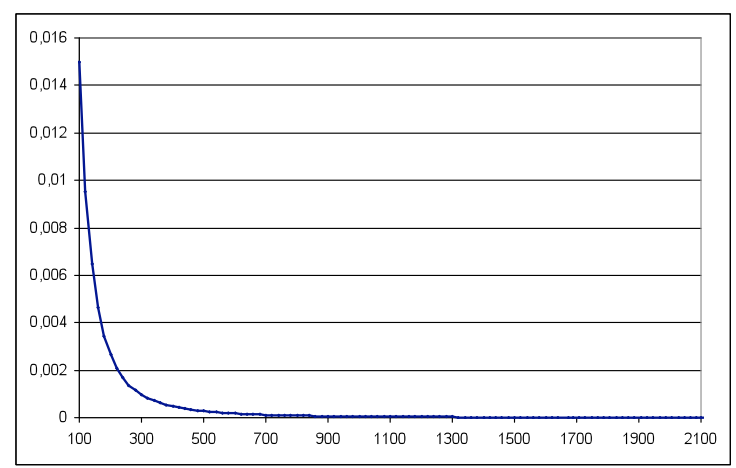

Ces résultats comparatifs ne changent pas quand le revenu minimum $h=100$ est modifié. Pareil avec le quotient $\frac{x(0,95)}{\mu}$.

Comme le signale Marc Barbut, quand $\alpha>1$ augmente, alors l'inégalité diminue, car le quantile $x(0,95)$ s'approche de la moyenne de la population. Or, nous avons vu que $\frac{x(0,95)}{\mu}=1$ dans l'exemple I, le concept antérieur d'inégalité n'est pas applicable parce que quand $\alpha \rightarrow \tilde{\alpha}$, avec $\alpha>\tilde{\alpha}$ et $\alpha<\alpha_{\max }$, l'inégalité diminue, et quand $\alpha \rightarrow \infty$, avec $\alpha>\alpha_{\max }$, l'inégalité diminue également.

\section{BIBLIOGRAPHIE}

BARBUT M. (1984), « Notes sur quelques indicateurs globaux de l'inégalité : C.Gini, V. Pareto, P.Lévy », Revue Française de Sociologie XXV, p. 609-622.

BARBUT M. (1998), «Une famille de distributions: des parétiennes aux antiparétiennes. Applications à l'étude de la concentration urbaine et son évolution », Mathématiques et Sciences humaines 141, p. 43-72. BARBUT M. (2003a), « Homme moyen ou homme extrême : de Vilfredo Pareto (1896) à Paul Lévy (1936) en passant par Maurice Fréchet et quelques autres », Journal de la Société française de Statistique, tome $144, \mathrm{n}^{\circ} 1-2$.

BARbUT M. (2003b), "Ideología, Matemáticas y Ciencias Sociales : V. Pareto, G. Sorel y la ambigüedad en la comparación de las desigualdades", Empiria 6, p. 11-29.

BARBUT M. (2007), La mesure des inégalités. Ambigüités et paradoxes, Genève-Paris, Librairie Droz.

BASUlto J., BUSTO J.J. (2010), “Gini’s Concentration Ratio (1908-1914)”, Journ@l Électronique d'Histoire des Probabilités et de la Statistique, vol. 6, n 1, Juin.

BASUlto J., BUSTO J.J., SANCHEZ R. (2011), "El concepto de desigualdad en Vifredo Pareto (1848.1923)", Capítulo 4, en Historia de la Probabilidad y la Estadística (V), J. M. Riobóo e I. Riobóo (editores). 
BOWLEY A.L. (1914), “The British Super-tax and the Distribution of Income”, Quarterly Journal of Economics 28, February, pp. 255-268.

BRESCIANI-TURRONI C. (1910), "Di un indice misuratore della disugualianza nella distribuzione della richezza", in Studi in onore di Baigio Bruginel XXX anno del suoinsegnamento, Palermo, Stab. Tip. Ditta L. Gaipa-Editore, p. 793-812.

CHAMBER J.M., MALLOWS C.L., STUCK B.W. (1976), "A Method for Simulation Stable Random Variables", Journal of the American Statistical Association, volume 71, n 354, June, pp. 340-344.

CHAMPERNOWNE D.G. (1953), “A Model of Income Distribution”, Economic Journal 63, June, pp. 318-351.

CHIPMAN J.S. (1976), “The Paretian Heritage”, Revue européenne des sciences sociales, vol. 14, $\mathrm{n}^{\circ} 37$, June, p. 65-173.

D’ADDARIO R. (1949), "Ricerche sulla curva dei redditi”, Giornale degli Economisti e Annali di Economia 8(1-2), pp. 91-144.

EDGEWORTH F.Y. (1898), "On the Representation of Statistics by Mathematical Formulae (Part I.)" Journal of the Royal Statistical Society, Vol. 81, n 4, Dec, pp. 670-700.

FELLER W. (1966), An Introduction to Probability Theory and Its Applications, Vol. 2, John Wiley and Sons, Inc.

FRÉCHET M. (1939), «Sur les formules de répartition des revenus », Revue de l'Institut international de statistique 3, p. 321-338.

LÉVY P. (1937), Théorie de L'addition des variables aléatoires, Paris, Gauthier-Villars.

SOREL G. (1897), « La loi des revenus », Le devenir social, $3^{\mathrm{e}}$ année, $\mathrm{n}^{\mathrm{o}}$ 7, p. 578-607. 